\title{
Fenomenología: filosofía comprensiva e interpretativa para el cuidado de enfermería
}

\author{
Acevedo-Figueroa, Lucila ${ }^{1 *}$; Álvarez-Aguirre, Alicia²
}

\begin{abstract}
RESUMEN
Introducción: El pensar para enfermería, es descubrir o redescubrir el sentido de aquello que llamamos, concepto, procedimiento, es fundar lo que se manifiesta fenomenológicamente buscando la esencia de la forma. Reflexionar respecto a la perspectiva fenomenológica en el proceso del cuidado incide en una práctica enfermera significativa y enriquecedora.Objetivo: Mostrar la filosofía fenomenológica a través de una mirada asistencial comprensiva e interpretativa, que describa la importancia de incluirla en el cuidado de enfermería encaminado a la comprensión del ser. Desarrollo: Comprender a la persona en situación de enfermedad en su dimensión humana utilizando el análisis reflexivo establece una forma de conocer la realidad, adentrándose en la esfera interna de las cosas para captar su verdadero sentido olvidando lo externo, lo evidente, lo que parte de una causa efecto, teniendo una estructura científica para interpretar y comprender, lo subjetivo del pensamiento, descubriendo estructuras esenciales de la conciencia y estableciendo la responsabilidad social de tiene enfermería, para el desarrollo de potencialidades, capacidades y posibilidades de bienestar en los individuos y la comunidad. Conclusiones: Los caminos para la atención de enfermería no terminan, mostrar la filosofía fenomenológica proporciona elementos para comprender que establece el ser humano con el entorno en la búsqueda de su salud, a partir de una mayor comprensión sobre la existencia humana y del ser enfermero.
\end{abstract}

Palabras clave: Filosofía; enfermería; investigación metodológica en enfermería; investigación cualitativa. (DeCS; BIREME).

${ }^{1 *}$ Estudiante de Doctorado en Ciencias de Enfermeria Campus Celaya- Salvatierra División de Ciencias de la Salud e Ingenierías de la Universidad de Guanajuato. Celaya Guanajuato, México. E-mail: acevedo_lucy@hotmail.com

${ }^{2}$ Doctora en Ciencias de Enfermería Departamento de Enfermería Clínica División de Ciencias de la Salud e Ingenierías Campus Celaya-Salvatierra Universidad de Guanajuato, México E-mail: alicia.alvarez@ugto.mx

*Autor para correspondencia

Cómo citar este artículo:

Acevedo-Figueroa L, Álvarez-Aguirre A. SFenomenología: filosofía compresiva e interpretativa para el cuidado de enfermería.Sanus. 2018;3(7): 6877. [Citado el _____ ]; Disponible en: mes día año URL 


\section{INTRODUCCIÓN}

Cada encuentro con el cuidado es único, es una experiencia subjetiva, una interacción con aspectos no visibles, en donde el acercamiento no se limita a referirse y aludir a alguien, si no que ella es la respuesta a algo, esta experiencia aún muda habrá de llevar a la expresión pura del propio sentido.

Parse, sostenía que las personas no se deben dividir en esferas porque el individuo es más que la suma de estas y enfermería orienta su mirada a la experiencia vivida por él, también señala que la relación entre la enfermera y el paciente surge al tratarse ambos como personas evitando crear juicios y etiquetas ${ }^{(1)}$.

Para lograr esta comprensión, la fenomenología redefine el conocimiento y modos de relación efectiva entre sujeto y objeto para mostrar la experiencia vivida incluyendo el significado y comprensión de las mismas ${ }^{(2)}$.

La autorreflexión, permite hacer cambios en el actuar de enfermería hacia una práctica reflexiva ${ }^{(3)}$. Es una herramienta para retomar el cuidado con encuentros intersubjetivos holísticos partiendo de diálogos abiertos y significativos donde a partir de la interacción, esta forma de estar frente al ser que necesita cuidados, enfermería reconoce los valores y prioridades diferentes a las propias generando a su vez nuevas oportunidades de crecimiento a la par con el sujeto ${ }^{(4)}$.

El paciente, en las unidades donde se proporciona cuidado de enfermería, es un ente pasivo quien recibe procedimientos, tratamientos y consejos estandarizados, unidireccionales de la institución, caracterizados por no enfocar al ser como alguien que está inserto en un entorno, tiempo y espacio que lo determina y que a su vez, él define y modifica con su presencia.

La fenomenología, puede ser ese hilo conductor del pensamiento que a permita un análisis en donde la razón no se separe de la emoción, ni el sujeto del objeto permitiendo a ambos seres reconocer su existencia y su fragilidad(5). $^{(2)}$.

Por lo que el propósito de este artículo es reflexionar sobre la filosofía comprensiva e interpretativa para el cuidado de enfermería.

\section{DESARROLLO}

Los orígenes de nuestras ideas y las múltiples interrogantes que enfermería se hace en su labor diaria, no se pueden resolver solo con indagación científica Kikuchi(6), menciona que la desviación en la interpretación de la ciencia por parte de las enfermeras y sus consecuencias derivadas, persistirán a menos que la filosofía como medio de indagación tome el lugar correspondiente en el mundo de enfermería, ya que, solamente filosofando, se puede establecer cuál cuestionamiento es susceptible de investigación científica y cuál no.

\section{Indagación filosófica}

La indagación filosófica, inicia de lo más abstracto encontrando el sentido de las cosas mismas, a la luz de las causas primarias, en tanto la indagación científica analiza objetos de una sola esfera del ser que investiga principios secundarios, así mismo tiene responsabilidad y obligación de dar forma a la cultura porque se sustenta en el sentido común.

Así mismo contribuye al conocimiento relacionado con lo que es, lo que pasa alrededor, con lo que el hombre busca y debe hacer. El elemento común entre la indagación filosófica y la científica es su dependencia en la experiencia para el desarrollo de conceptos, formulación de cuestionamientos y la prueba de proposiciones y teorías ${ }^{(7)}$.

La filosofía es discursiva, depende de la experiencia común, no se puede demostrar su falsedad o veracidad apelando a ninguna clase de experiencia. llumina sobre las últimas causas, resuelve conflictos entre disciplinas, además puede guiar las conductas individuales y sociales.

La filosofía es la "búsqueda del saber". Por sus raíces etimológicas es "amor por la sabiduría", investiga las causas y leyes que subyacen a la realidad. Las ramas que la conforman, entre otras, son: lógica, ética, estética, epistemología, metafísica, axiología, política. La filosofía se ocupa de comprender la verdad, razonamiento y realidad de los seres humanos y determina la forma correcta de vivir. Enfermería se beneficia de la filosofía, ya que ella rige sus métodos a través de la lógica y la ética ${ }^{(8)}$.

La indagación filosófica para enfermería permite ser crítico y sistematizar el conocimiento surgido de la investigación empírica, práctica clínica, aprendizaje y hasta de la misma intuición. La filosofía es relevante para enfermería, ambas comparten un sentido humanístico en su quehacer, integran la diversidad de pensamiento. Por ejemplo, crear ciencia de enfermería a través del conocimiento tácito y comunicarla por conocimiento explícito $^{(9)}$.

La naturaleza, de manera integral provee unidad a las formas de conocimiento que guían a la interpretación holística del fenómeno central de enfermería. En esa comprensión, enfermería se mueve mas allá de percepciones estereotipadas, esto capacita el entender lo escondido y real a través de la fenomenología, abriendo a una nueva concepción del "ser" humano.

\section{Fenomenología}

La palabra fenomenología desciende del griego "fenomenon" que denota mostrarse a sí misma y "logos" representa discurso o ciencia. Se comprende como ciencia que trata sobre cómo revelar y hacer visibles las realidades a través de la experiencia subjetiva, en intentar encontrar las razones y no las causas de los hechos 
para lograr el conocimiento en la experiencia vivida y regresando a las cosas mismas para mirarlas tal como ellas se manifiestan ${ }^{(10)}$.

Enfermería en la búsqueda de la univocidad del fenómeno (su idealidad), trae como lenguaje precepciones de la experiencia humana con todo tipo de fenómenos. La filosofía del cuidado en enfermería puede orientarse hacia dos vertientes: tener una visión mecanicista que refleja reactividad, prioriza el conocimiento o bien se centra en el ser y sus expectativas, que le permiten comprender, interpretar y encontrar un significado de las situaciones que vive. La libertad, entendida como elección y responsabilidad, se concibe como un proceso de tensión que genera desarrollo en la profesión ${ }^{(11)}$.

La disciplina de enfermería es humana y la fenomenología se acerca a lo propiamente humano, ambas producen conocimiento científico que permite fortalecer la práctica de enfermería, por lo tanto, contribuye a su mismo desarrollo.

El nuevo orden epistemológico no considera al sujeto $\mathrm{y}$ al objeto independiente uno del otro sino vinculados por el acto cognoscitivo. Este abordaje permite que se interpreten los fenómenos emanados desde la subjetividad de las personas y el mundo que les rodea en busca de la esencia del propio ser y los procesos.

La fenomenología y su carácter subjetivo, está ligado a la esencia del mismo ser humano, permitiéndole acceder a diversas esferas del ser cuidado, que es parte del tiempo y espacio en el mundo. El enfoque fenomenológico visualiza la realidad que se conoce con el mundo- cuidado a través de experiencias ${ }^{(12)}$.

Esta relación aporta un intercambio, el sujeto, vivencias y objeto, el marco del cual existe como ente. Este enfoque busca redefinir las formas de relación efectiva entre sujeto, objeto que tienen lugar para proclamar los contenidos fenomenológicos. Estos fenómenos se describen de manera consiente sin teorías sin causalidades libres de supuestos y prejuicios.

La conciencia en fenomenología es el estar vuelto para el atento. Mostrarse o exponerse a la luz, sin obscuridad, no ocurre en una primera mirada al fenómeno, pero poco a poco se da en la búsqueda atenta y rigurosa del sujeto que interroga y que busca ver más allá de la apariencia, insistiendo en la búsqueda de lo característico, básico, esencial del fenómeno, es decir, aquello que se muestra para el sujeto ${ }^{(13)}$. El paciente es más que sus datos biológicos, también es biografía, no solo padece una enfermedad, sino que también tiene temores y expectativas, un modo de ser vida.

En la persona a quien se le proporciona cuidado, que puede encontrarse en pasillos fríos, esperas largas, diagnósticos confusos, se debe considerar y retomar lo que Husserl propone "volver a las cosas mismas", ayudaría a comprender la intencionalidad, idea principal de la fenomenología expresada como carácter sustancial de la conciencia que sucede en la interacción con enfermería en el momento de cuidado.

\section{Cuidado}

El proporcionar cuidado transcurre desde la acción hasta el ser, construido a partir de experiencia profesional, que alberga características especiales cuando es proporcionado. El cuidado se define como actividad con valor personal y profesional dirigido a la conservación, restablecimiento y autocuidado de la vida basado en la relación enfermera-paciente.

Collière ${ }^{(14)}$, enfermera antropóloga y filósofa, expresa que "Cuidar es un acto de vida cuyo objeto es, por encima de todo, permitir que la vida continúe y se desarrolle y de ese modo luchar contra la muerte del individuo, del grupo y de la especie". Asimismo, comprender el cuidado de enfermería a partir del contexto o del proceso vida y muerte al que el ser humano se enfrenta diariamente.

El proporcionar cuidado, como enfermera compromete al autoconocimiento y actividades cognitivas expresadas en el momento de cuidado por medio de análisis, reflexión, valoración, intuición, decisión y la sensibilidad.

El profesional de enfermería aprecia a la persona cuidada como una unidad, destacando la interacción persona-contexto como una evolución recíproca que trascienden en la relación persona-enfermera y las limitaciones de tiempo y espacio, donde ambos se desarrollan ${ }^{(15)}$.

Esta visión, nos permite situarnos frente al otro con un tipo de comprensión de orden superior, por una intuición reflexiva para escribir y clarificar la experiencia tal como la viven la persona que cuida y la persona que requiere cuidado(16).

\section{CONCLUSIONES}

La fenomenología permite a enfermería tener una visión de la persona cuidada cómo única, diferente, irreducible, que responden simultáneamente a estímulos y autoorganizada por comportamientos, cualidades y características del entorno; en un contexto específico en el que se consideran las creencias, las experiencias subjetivas, las ideologías, la intuición entre otros. Además de brindar respuestas a las preguntas relacionadas con las situaciones que son experimentadas como parte del cuidado de la experiencia de la salud humana.

El cuidado desde la fenomenología se interpreta como un proceso organizado que evoluciona a través de la historia. Es holístico, no fracciona el cuerpo, sale de las explicaciones causales, simplistas, considera el contexto. La fenomenología en el encuentro de cuidado permite reconoce a los actores a fin de comprender y significar la realidad. A través de las vivencias se exploran la palabra, la relación intersubjetiva y el diálogo que favorecen la construcción de una relación comprensiva de cuidado ${ }^{(17)}$. En este sentido el cuidado desde el enfoque 
fenomenológico incluye intervenciones de enfermería para y con el ser, fundamentadas en conocimiento y habilidades que promuevan, mantengan y recuperen la totalidad del ser. Llama a reflexionar sobre su vivencia e interpretar la práctica para comprensión del ser cuidado. Recalca que el cuidado debe ser renovado cada vez, porque el ser humano que cuidamos no es un objeto sin emociones, sino un ser que sufre y que tiene necesidad de ayuda para madurar y volver a encontrar el sentido de su vida.

La reflexión aquí realizada nos confirma la visión holística que debe desarrollar enfermería en el abordaje del cuidado de la persona. Enfermería en la actualidad avanza en múltiples aspectos técnicos y de procesos para mejorar el cuidado, sin embargo, se quedan sin un sentido radical para ambos seres.

A nivel asistencial, existe dualidad de lo que demandan las personas y la oferta de los servicios como disciplina de enfermería. Los hábitos y rutinas que ha creado enfermería quedan demasiado lejos de la esencia propia de enfermería, por lo que incluir a la fenomenología como un saber comprensivo e interpretativo ayuda a explicar la estructura o esencia de una experiencia vivida en la búsqueda de la interacción de ambas personas, en el cuidado de enfermería. La hoja de registros clínicos es para crear evidencia del cuidado, en donde la descripción precisa a través de la experiencia vivida se visualice y por ende se percibe en ambos seres.

Coincidimos en considerar que la fenomenología es la forma de llegar a la esencia de los fenómenos de estudio, sí, apegado de ella le devuelve al paciente su verdadera humanidad, será una tarea que dejará satisfacción por lo realizado y a su vez lo recibido, tarea a la que estamos requeridos todos, frente a la exigencia que no solo concierne, a la fenomenología si no al quehacer filosófico en general, lo que sería un gran paso en la comprensión de esos silencios cuando la persona es visitada por la enfermedad.

\section{REFERENCIAS BIBLIOGRAFICAS}

1. Parse RR. Man-Living-Health: A Theory of Nursing. New York:Wiley; 1981.

2. Fernandes $M$, Merighi $M A B$, Muñoz LA. La enfermera clínica y las relaciones con los estudiantes de enfermería: un estudio fenomenológico. Texto Contexto Enferm [internet]. 2010 Sep[citado 2018 Ago 14];19(1): 112119. Disponible en: http://www.redalyc.org/articulo. oa?id=71413596013

3. Minguez I, Siles J. Pensamiento crítico en enfermería: de la racionalidad técnica a la práctica reflexiva. Aquichan [Internet]. 2014 [citado 2018 Jul 15];14(4):594604. Disponible en: http://www.redalyc.org/articulo. oa? id $=74133057013$
4. Chocarro L. La intersubjetividad y su relevancia en los cuidados de Enfermería. Index Enferm [Internet]. 2013 Dic [citado 2018 Ago 05]; 22(4): 219-221. Disponible en: http://dx.doi.org/10.4321/S1132-12962013000300006

5. Nogales A. Cuidados de Enfermería en el siglo XXI: una mirada hacia el arte de cuidar. Cultura de los cuidados [Internet]. 2012 Jun [citado 2018 Julio 22]; 0(31): 41-55. Disponible en: https://doi.org/10.7184/cuid.2011.31.06

6. Kikuchi JF. Nursing Questions that Science Cannot Answer. In Philosophic Inquiry in Nursing. Newbury Park, Ca: Sage Publications; 1992. pp. 26-37.

7. Simmons H. Philosophic and Scientific Inquiry: The Interfase. In Kikuchi, IE, Simmons H. Philosophic Inquiry in Nursing. Newbury Park, Ca: Sage Publications; 1992. pp. 9-25.

8. Diaz MG. Filosofía de la ciencia del cuidado. Analogía del mito de la caverna de Platón con la profesión de enfermería. Rev. Iberoamericana de Ciencias de la Salud. [Internet].2013 Jun [citada 2017 Junio15]; 2(3): 1-15. Disponible en: https://www.rics.org.mx/index.php/RICS/ article/view/17/22

9. Sánchez B, Pinto N. Dimensiones del Cuidado. Grupo de Cuidado. Bogotá Colombia: Unibiblos; 1998. 46-71 p.

10. Barbera N, Inciarte A. Fenomenología y hermenéutica: dos perspectivas para estudiar las ciencias sociales y humanas. Multiciencias [Internet]. 2012 Jun [citada 2018 Ago 21]; 12(2): 199-205. Disponible en: http://www. redalyc.org/articulo.oa?id $=90424216010$

11. Acebedo S, Rodero V, Vives C, Aguarón MJ. La mirada de Watson, Parse y Benner para el análisis complejo y la buena práctica. Index Enferm [Internet]. 2007 Abr [citada 2017 Ago 05]; 16(56):40-4 Disponible en: $\quad$ http://scielo.isciii.es/scielo.php?script=sci_ arttext\&pid=S113212962007000100009\&lng =s

12. Muñoz LA, Erdmann AL. Metodología de la investigación fenomenológica en enfermería. En: Do Prado ML, De Souza M, Monticelli M, Cornetto MC, Gómes PF editores. Investigación cualitativa en enfermería. Metodología y didáctica. Washington: Organización Panamericana de la Salud OPS; 2013. 12-22 p.

13. Do Prado ML, De Souza M, Monticelli M, Cometto MC, Gómes PF. Investigación en enfermería. Metodología y didáctica. Washington: Organización Panamericana de la Salud OPS; 2013. 276 p.

14. Collière MF. Promover la vida. México: Interamericana McGraw-Hill; 2003.392 p. 
15. Durán MM. Marco epistemológico de la enfermería. Aquichán. [Internet] 2002 Dic [Citado 2018 Agosto 01]; 2(1): 7-18. Disponible en: http://www.scielo.org.co/scielo.php?script =sci_ arttext\&pid=S165759972002000100003\&lng=en

16. Rodríguez $\mathrm{S}$, Cárdenas $\mathrm{M}$, Pacheco $\mathrm{AL}$, Ramírez M. Una mirada fenomenológica del cuidado de enfermería. Enferm. univ [ Internet]. 2014 Dic [citado 2018 Jul 06]; 11 (4): 145-153. Disponible en: http://www.scielo.org.mx/scielo.php?script =sci_ arttext\&pid=S166570632014000400005\&lng=es

17.Ramírez M, Cárdenas M, Rodríguez S. El Dasein de los cuidados desde la fenomenología hermenéutica de Martín Heidegger. Enferm. univ [Internet]. 2015 Jul [Citado15 Ago 2018]; 12(3): 144-151. Disponible en: http://dx.doi.org/10.1016/j.reu.2015.07.003. 